\title{
Validation of a measurement method for magnetic shielding effectiveness of a wire mesh enclosure with comparison to an analytical model
}

\author{
M. Kühn ${ }^{1}$, W. John ${ }^{2}$, and R. Weigel ${ }^{3}$ \\ ${ }^{1}$ AUDI AG, 85045 Ingolstadt Germany \\ ${ }^{2}$ SIL System Integration Laboratory GMBH, Technologiepark 32, 33100 Paderborn Germany \\ ${ }^{3}$ Lehrstuhl für Technische Elektronik, Friedrich-Alexander-Universität Erlangen-Nürnberg, Cauerstrasse 9, \\ 91058 Erlangen Germany \\ Correspondence to: M. Kühn (michael.kuehn@audi.de)
}

\begin{abstract}
This paper deals with the validation of a measurement method for determining the magnetic shielding effectiveness of a wire mesh enclosure in the frequency range from $10 \mathrm{kHz}$ to $150 \mathrm{kHz}$. The comparison with an analytical model (parallel-plate-shield, see Kaden, 1959) for magnetic shielding effectiveness of wire mesh is also part of this contribution. To measure the shielding effectiveness of an enclosure in general, two steps are necessary:
\end{abstract}

1. Reference measurement of the incident electro-magnetic field.

2. Measurement of the incident electro-magnetic field in the enclosure by same conditions.

This method presented in the contribution uses a Helmholtz coil as magnetic field source, controlled by a signal generator and an amplifier in voltage mode. At first, a field meter was used to measure the frequency dependent magnetic field in the center of the Helmholtz coil. The magnetic field strength was also analytically calculated and compared to the measurement results. The next step was to measure the induction voltage caused by the magnetic field with a magnetic field probe, which was later used for the measurements of the shielding effectiveness either. A signal amplifier was needed to raise the signal-to-noise-ratio (SNR). The gain of the signal amplifier was also determined and measured. Two positions of the enclosure in the Helmholtz coil were considered, horizontal and vertical. The vertical position of the enclosure approaches the analytical model of Kaden (1959) closely and the results show a good match with the analytical model.

\section{Introduction}

Vehicles with electric drive have enormous potential to reduce the growing dependence on fossil fuels, for sustainable economic growth and to support environmentally-friendly energy usage. The German government has set a goal for Germany to be the market leader in the development of these technologies in the next years. However, huge technological challenges, which can only be addressed through an extraordinary degree of interdisciplinary research, are standing in the way of Germany achieving the above said goal. Apart from some individual solutions that represent a certain level of vehicle functionality, the requirements for a mass market product are immense. Customer demands in reference to driving safety, comfort and quality, over and above, the perseverance of electromagnetic compatibility (EMC) are obligatory requirements (such as CISPR 25) which need to be met. The key challenge here is the electro-magnetic decoupling of the electric powertrain, which is composed largely of coaxial cables, connectors, a battery, the inverter and the electric motor. Within the machine, a rotating field is generated through the pulse-width modulated (PWM) voltages of the inverter, which typically have fundamental frequencies between the range of $8 \mathrm{kHz}$ to $24 \mathrm{kHz}$, Michel (2011). Strong magnetic fields are the result with a wide spectrum up to the $\mathrm{MHz}$ range. The entire high-voltage wiring is performed in a completely closed shielding to comply with the requirements for electrical safety and the reduction interference emissions Hillmer et al. (2009). Conventional shielding against magnetic fields are made of solid metal plates with thicknesses from $1 \mathrm{~mm}$ upward, (Schwab and Kürner, 2010). 
Evidently, the resulting weight gain is counterproductive to an efficient hybrid or electric vehicle. Therefore, lightweight fabrics, composite materials or wire mesh screens come to the fore. For predicting the shielding effectiveness of those materials it is necessary to validate measurement methods and their results with analytical models.

\section{Magnetic shielding effectiveness}

The magnetic shielding effectiveness is a part of the shielding effectiveness in the frequency domain. The frequency range is divided in two parts of shielding effectiveness. These are magnetic shielding effectiveness and electromagnetic shielding effectiveness. The magnetic shielding effectiveness represents the behaviour of the shield in the frequency range where the wavelength is large compared to the dimensions of the shield or enclosure. The magnetic field will be reduced by two physical processes (see Fig. 1), the generation of eddy currents in the shield and skin effect. The incident magnetic field leads to a voltage in the shield, which results in eddy currents in the shield. These eddy currents are generating an inverse oriented magnetic field, which weakens the incident magnetic field. The skin effect describes the reduction of the current density of a conductor or shield by raising frequency. In summary, the magnetic shielding effectiveness depends on the shield material $\left(\sigma, \mu_{r}\right)$ and the thickness of the shield. In the frequency range to be considered here, the incident electrical field could be neglected due to Faraday cage effect. When the wavelength reaches the dimensions of the shield or enclosure (approximately $\lambda / 10$, see also Schwab and Kürner, 2010) the electromagnetical shielding effectiveness gets dominant. The shielding effectiveness is influenced by apertures and the shield dimensions itself. Therefore, resonances occur at discrete frequencies, which are represented by a drop in shielding effectiveness. The complete shielding behaviour in the frequency range is shown in Fig. 1, (Wolfsperger, 2008).

Two analytical models, based on a parallel-plate-shield from Kaden (1959), for calculating the magnetic shielding effectiveness in the frequency range of a homogeneous material and a wire mesh will be used for comparison.

\section{State of the art}

This chapter describes two analytical models selected from the literature. The main focus was laid on analytical models for calculating magnetic shielding effectiveness of enclosures. As above described, the wavelength is large compared to the dimensions of the enclosure or shield. Two major models from Kaden (Kaden, 1959) are described for a homogeneous material (for example: copper, aluminum) and a wire mesh screen. There are other models available for calculating the coupling through the apertures of the mesh, for example Casey (1988) or Wolfsperger (2008).

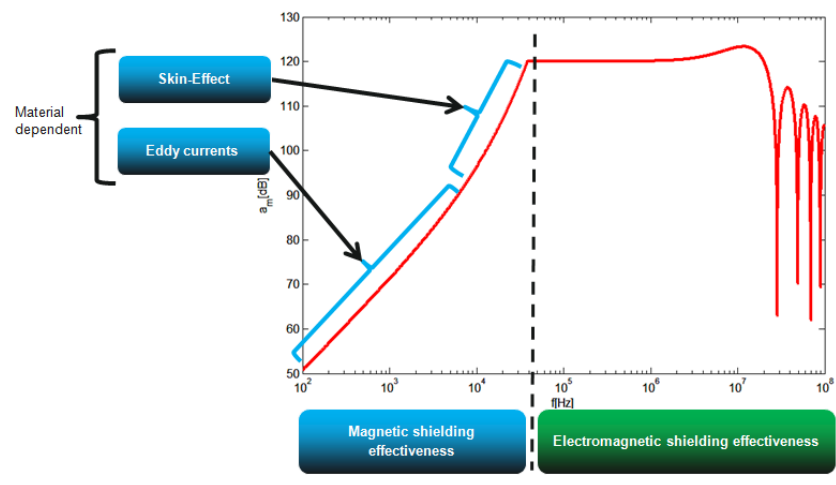

Fig. 1. Principle behaviour of shielding effectiveness $a_{m}[\mathrm{~dB}]$ in the frequency domain.

\subsection{Analytical model for homogeneous materials}

At first we analyse the magnetic shielding effectiveness of a parallel-plate-shield based on homogeneous material defined by a model from Kaden (1959). The parallel-plate-shield represents a closed shield structure (like a conductor loop) with the condition, that the distance between the plates is even smaller than the length of the plates $\left(|y|>>x_{0}\right.$, see Fig. 2). The model is based on the ratio of the magnetic field component inside and outside of the parallel-plate-shield. This model is only valid for the magnetic shielding effectiveness, so the wavelength is even larger than the screen dimensions. The skin effect is also considered by this model. The magnetic shielding effectiveness is calculated by Eq. (1) and (2) for a parallel oriented vector of the magnetic field to the parallel-plate-shield, so the incident field penetrates the conductor loop (Kaden (1959), page 74, equation 11, 12).

$K=\frac{k_{w} \cdot x_{0}}{\mu_{r}}$

$a_{s}=20 \cdot \log \left|\cosh \left(k_{w} d\right)+K \sinh \left(k_{w} d\right)\right|$

The parameter $k_{w}$ describes the eddy current constant, $x_{0}$ is the half distance between the plates and $d$ is the shield thickness. The main parameters for influencing the shielding effectiveness are the conductivity $(\sigma)$, the permeability $\left(\mu_{r}\right)$, both considered in the eddy current constant $k_{w}$, and the shield geometry. Figure 3 shows the magnetic shielding effectiveness of a parallel-plate-shield (copper and aluminum, $x_{0}=0.15 \mathrm{~m}, d=1 \mathrm{~mm}$ ) with respect to frequency.

Figure 3 describes in the frequency domain $(100 \mathrm{~Hz} \leq$ $f \leq 1 \mathrm{MHz}$ ) the behaviour of the shielding effectiveness of a parallel-plate-shield structure penetrated by a magnetic field. The shielding effectiveness was limited to $120 \mathrm{~dB}$, because higher values are not realistic and the electromagnetic shielding effectiveness was also not considered. Theoretically the 


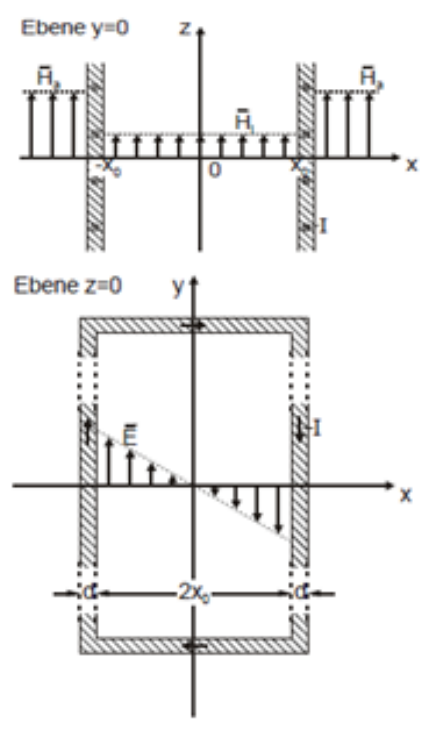

Fig. 2. Structure of a parallel-plate-shield for homogeneous materials (Kaden, 1959).

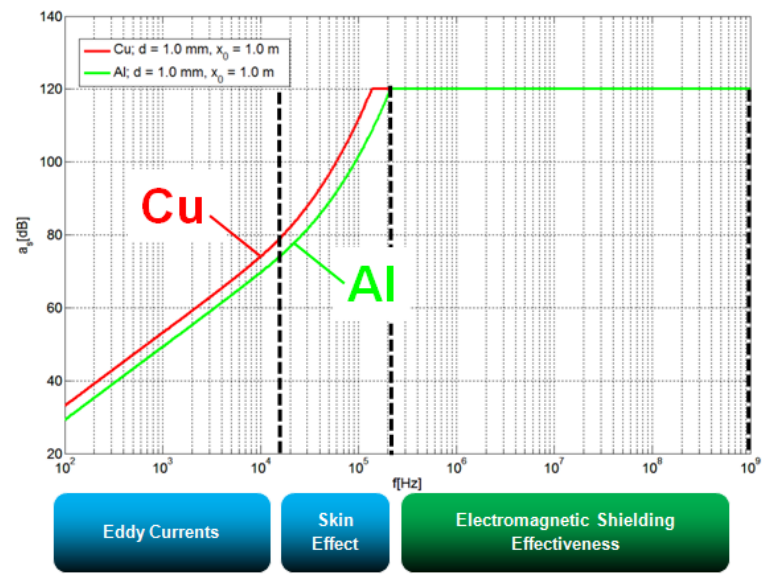

Fig. 3. Calculated shielding effectiveness of a parallel-plate-screen using the Kaden model.

magnetic shielding effectiveness would converge to infinity by raising frequency. Figure 3 shows also, that the magnetic shielding effectiveness of the copper parallel-plate-shield is approximately $3 \mathrm{~dB}$ higher than the shielding effectiveness of the same structure build by aluminum material. Another model of the parallel-plate-shield for homogeneous materials from Kistenmacher et al. (1995) is available and similar to the above described model from Kaden. The main difference is the consideration of the area and the circumference (ratio) instead of the distance between the plates.
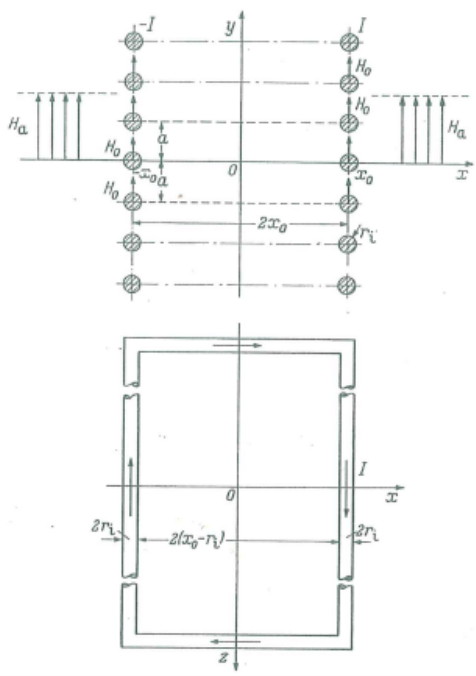

Fig. 4. Structure of a parallel-plate-shield based on wire mesh (Kaden, 1959).

\subsection{Analytical model for wire mesh screens}

In order to describe the magnetic shielding effectiveness of a parallel-plate-shield based on a wire mesh, a model from Kaden (1959) will also be used for comparison of measurement results. In this case the homogeneous material is substituted by a wire mesh. The wire mesh to be investigated consists of equidistant wires, which are defined about the distance to each other (mesh size) and the wire radius. Figure 4 shows the model structure of the parallel-plate-shield for a wire mesh used for the analysis. The physical shielding effects are similar to the parallel-plate-shield for homogeneous materials. The difference is, that opposite wires are forming a short connection, where eddy currents are induced and generating an inverse directed magnetic field, which weakens the incident magnetic field. The variation of the wire resistance and inductance in dependency of the frequency is also, due to the skin effect, part of the Kaden model (Kaden (1959), page 276 , equation 16 ).

Figure 5 shows the calculated magnetic shielding effectiveness as a function of frequency. As in Fig. 3, two materials (copper and aluminum) are compared. The mesh size $\left(a_{s}\right)$ is $1 \mathrm{~mm}$ and the wire radius $\left(r_{w}\right)$ is $10 \mu \mathrm{m}$. As in Fig. 3 shown, copper has a higher magnetic shielding effectiveness in comparison to aluminum by the same shield conditions (approximately $4 \mathrm{~dB}$ ). This is reasoned by the higher conductivity of copper. The field penetration through the mesh is not considered, the model is only valid for the magnetic shielding effectiveness determination. (The characteristic parameters of the parallel-plate-shield model for wire mesh are: the conductivity $(\sigma)$, the permeability $\left(\mu_{r}\right)$, the distance between the plates $\left(x_{0}\right)$, the mesh size $\left(a_{s}\right)$ and the wire radius $\left.\left(r_{w}\right)\right)$. 


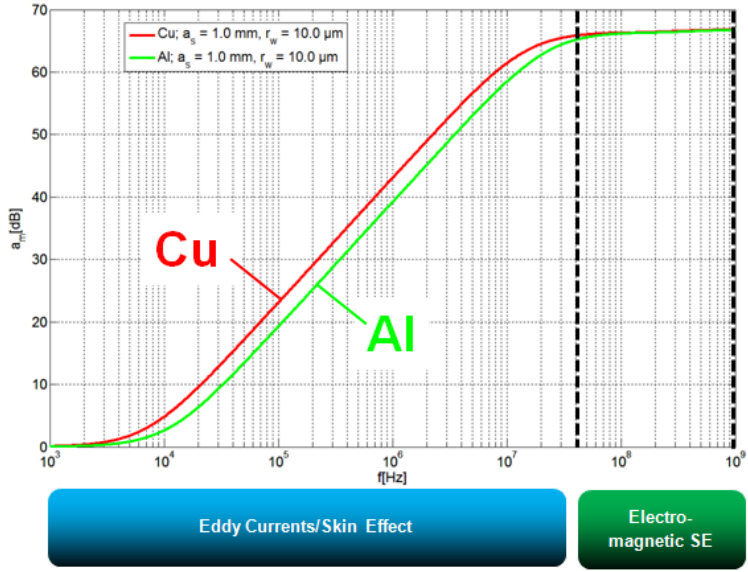

Fig. 5. Calculated shielding effectiveness of a wire mesh parallelplate-shield using the Kaden model.

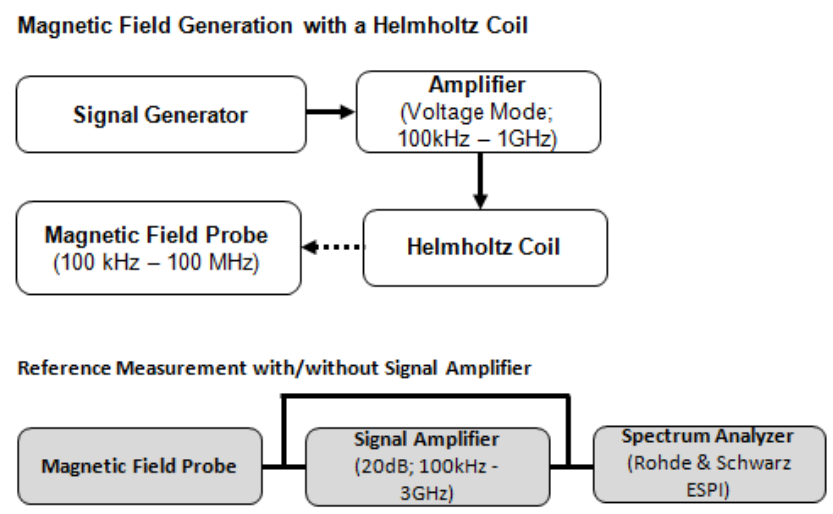

Shielded Measurement with an Horizontal/Vertical Oriented Enclosure

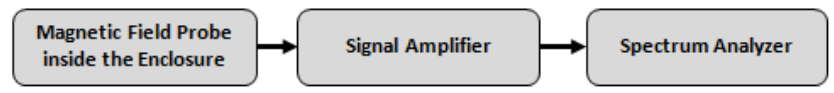

Fig. 6. Setup for shielding effectiveness measurements.

\section{Measurement setup}

This chapter describes the setup and the achieved results of the magnetic shielding effectiveness measurement. A Helmholtz coil was used to generate the magnetic field. The field generation was controlled by a signal generator and an amplifier in voltage mode. A constant amplitude $(4 \mathrm{~V}$, sine wave, peak-to-peak) in the frequency range from $10 \mathrm{kHz}$ to $150 \mathrm{kHz}$ was adjusted in the signal generator and boosted by the amplifier ( $40 \mathrm{~dB}$ voltage gain). Figure 6 shows a block diagram of the measurement setup for magnetic field generation by the Helmholtz coil, reference measurement and shielded measurement.

Consequently, there was no constant magnetic field amplitude. It was dependent on the impedance of the Helmholtz coil. The magnetic field in the center of the Helmholtz coil

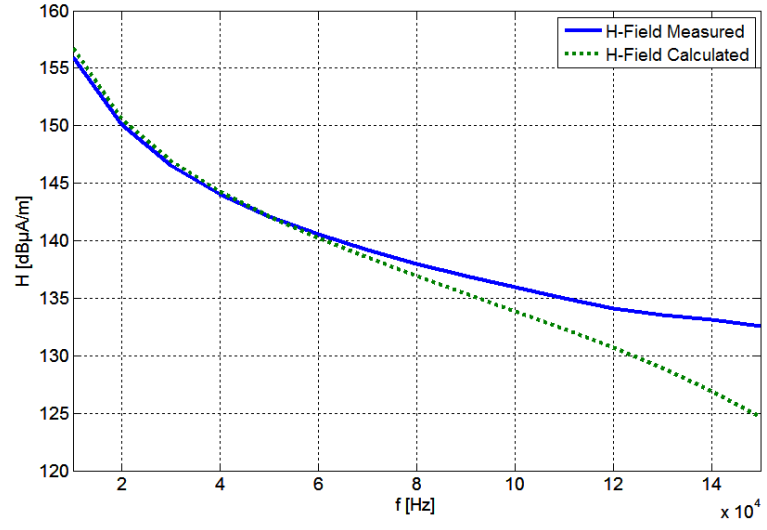

Fig. 7. Measured and calculated magnetic field in the center of the Helmholtz coil.

was measured with a field meter (solid line in Fig. 7) and analytically calculated (dotted line in Fig. 7). The difference of measured and calculated values occurs due to the complex resistance of the coil pair and the influence of the capacitive coupling between the layers of the coil. The complex resistance was calculated for this application with a simplified circuit model of the Helmholtz coil.

For the measurement of the induced voltage, a magnetic field probe from Rohde \& Schwarz $(6 \mathrm{~cm}$ diameter, specified frequency range: $100 \mathrm{kHz}$ to $100 \mathrm{MHz}$ ) and a signal amplifier from Langer EMV ( $20 \mathrm{~dB}$ gain, specified frequency range: $100 \mathrm{kHz}$ to $1 \mathrm{GHz}$ ) to raise the SNR were used. Additionally a spectrum analyzer also from Rohde \& Schwarz was applied. The signal amplifier was validated with measurements of the induced voltage with and without the signal amplifier. In Fig. 8 the induced voltage measured with and without the signal amplifier as a function of frequency is visualized. The graph shows, that the induction voltage for both measurements is nearly constant, except for the lower frequencies, where the signal amplifier does not work properly. By subtracting both curves, the gain of the signal amplifier could be calculated and for approximately $19 \mathrm{~dB}$ in the frequency range from $30 \mathrm{kHz}$ to $150 \mathrm{kHz}$.

With the magnetic field measurement results shown in Fig. 7 and the induced voltage shown in Fig. 8, the transfer function of the field probe can be calculated. For calculating the shielding effectiveness of the wire mesh enclosure, two measurements are necessary:

1. Reference measurement with magnetic field probe in the center of the Helmholtz coil.

2. Shielded measurement with magnetic field probe in the geometric center of the enclosure in the Helmholtz coil. center

The measurement procedure applied here is based on the standard DIN EN 61000-5-7. 


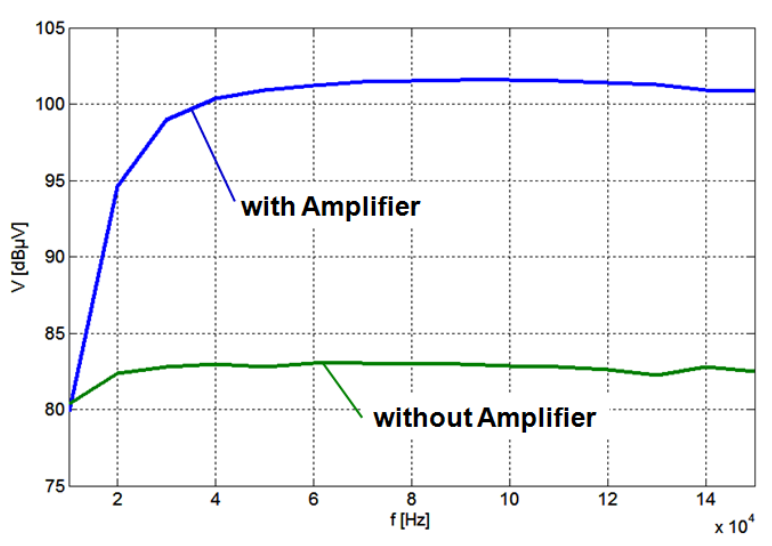

Fig. 8. Measured voltage of the field probe located in the center of the Helmholtz coil.

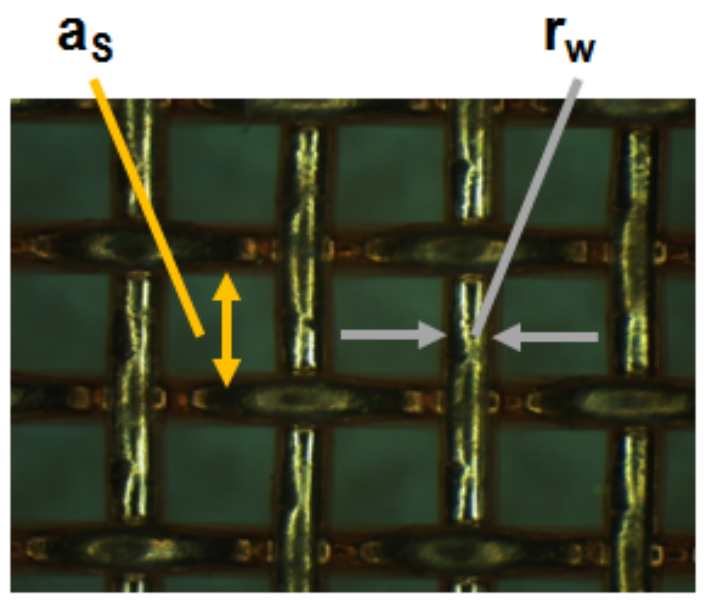

Fig. 9. Microscopic view of the wire mesh.

\subsection{Wire mesh enclosure}

For the magnetic shielding effectiveness measurements a wire mesh enclosure was chosen, too. The enclosure based on a wooden frame with overlapping copper wire mesh on each site for an electrical connection of the sides, so that a closed shield structure is guaranteed. The size of the enclosure is $30 \cdot 30 \cdot 10 \mathrm{~cm}$, the mesh material is copper with a mesh size of $\sim 364 \mu \mathrm{m}$ and wire radius of $\sim 100 \mu \mathrm{m}$, see microscopic view in Fig. 9. Two positions in the Helmholtz coil are possible for the enclosure: horizontal (leads to small areas for the incident field) and vertical (large areas for the incident field). The enclosure in the vertical position approaches the above introduced parallel-plate-shield model for wire mesh nearly. The following Fig. 10 shows the wire mesh enclosure with opened cover, the magnetic field probe was located in the geometrical center and was rotated to the field direction accordingly.

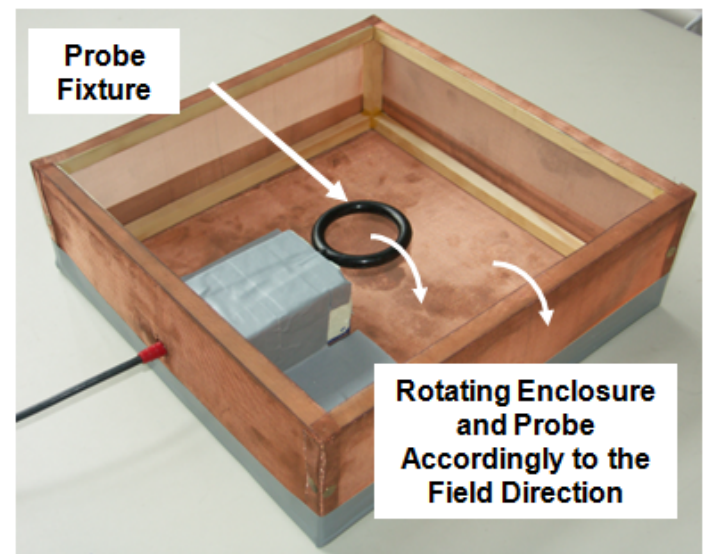

Fig. 10. Wire mesh enclosure and position of the magnetic field probe.
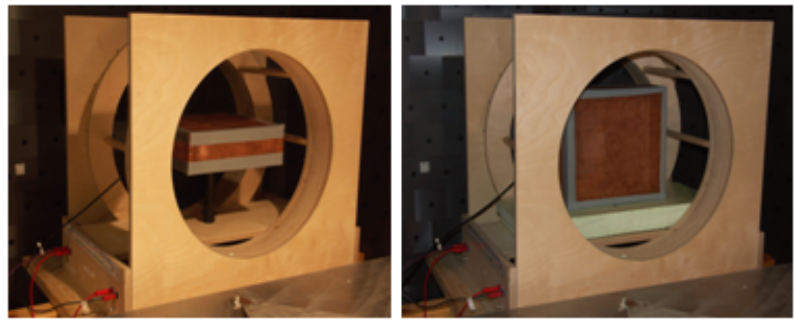

Fig. 11. Horizontal (left) and vertical (right) position of the enclosure inside the Helmholtz coil

For the shielded measurement horizontal and vertical positions of the enclosure were considered (see Fig. 11).

\section{Measurement results}

A discussion of the results and a comparison with the analytical model (Kaden (1959), page 276, equation 16) will be presented. The shielding effectiveness was calculated by subtracting the induced probe voltage values of the shielded measurement from the reference measurement. Equation 3 shows this relation and must be calculated for each frequency step. All units are in $\mathrm{dB}$ and depend on the frequency.

$\mathrm{SE}=V_{\text {ref }}-V_{\text {shield }}$

\subsection{Reference measurement of magnetic shielding effectiveness}

The reference measurement was done by placing the magnetic field probe in the center of the Helmholtz coil and measuring the induced voltage. The frequency depended induced voltage is shown in Fig. 8. As above described, the induced voltage falls about $20 \mathrm{~dB} \mu \mathrm{V}$ by $10 \mathrm{kHz}$, because the signal amplifier is not specified for the frequency range below $100 \mathrm{kHz}$, this does not affect the shielding effectiveness 


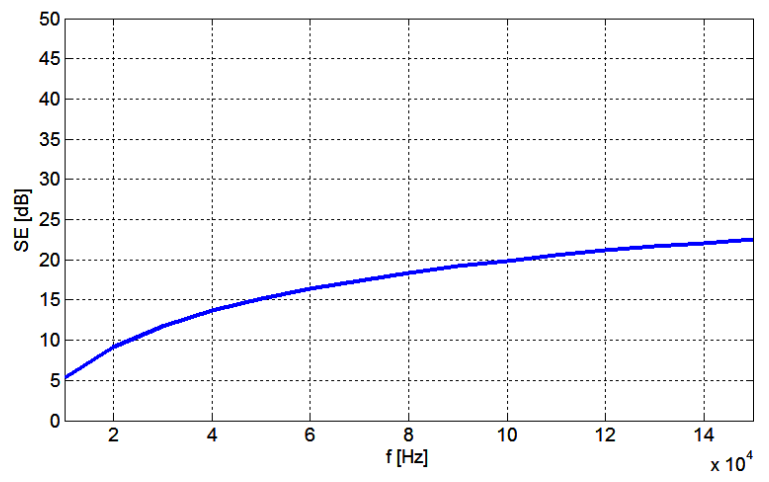

Fig. 12. Measurement result of the shielding effectiveness (SE) of a horizontal oriented wire mesh enclosure (see also Fig. 11 - left)

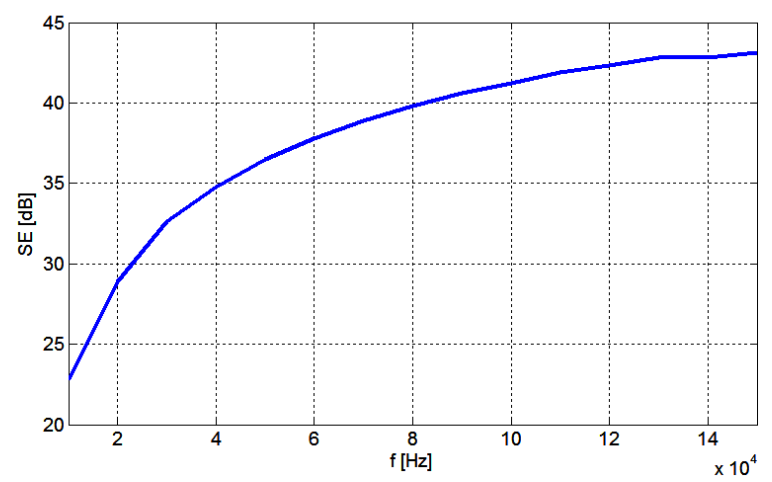

Fig. 13. Measurement result of the shielding effectiveness (SE) of a vertical oriented wire mesh enclosure (see also Fig. 11 - right)

measurement. Due to the fact that the conditions are the same for all measurements (with and without enclosure).

\subsection{Shielded measurement}

The results for the measurement in the horizontal position are shown in Fig. 12. The magnetic shielding effectiveness is very low (from $5 \mathrm{~dB}$ to $23 \mathrm{~dB}$ in the frequency range to be considered), this is reasoned by a small area for the incident field $(10 \cdot 30 \mathrm{~cm})$, so the propagation of eddy currents was limited. Therefore the compensation of the incident field caused by the inverse directed magnetic field of the eddy currents is very low.

The results for the measurement in the vertical position are shown in Fig. 12. The magnetic shielding effectiveness of the vertical position is in comparison with the results of the horizontal position larger $(\sim 20 \mathrm{~dB})$. This is due to larger area $(30 \mathrm{~cm} \cdot 30 \mathrm{~cm})$ for the incident field and the occurred eddy currents in field direction. Therefore, there was a better compensation of the incident field by inverse directed magnetic field resulting from the eddy currents.

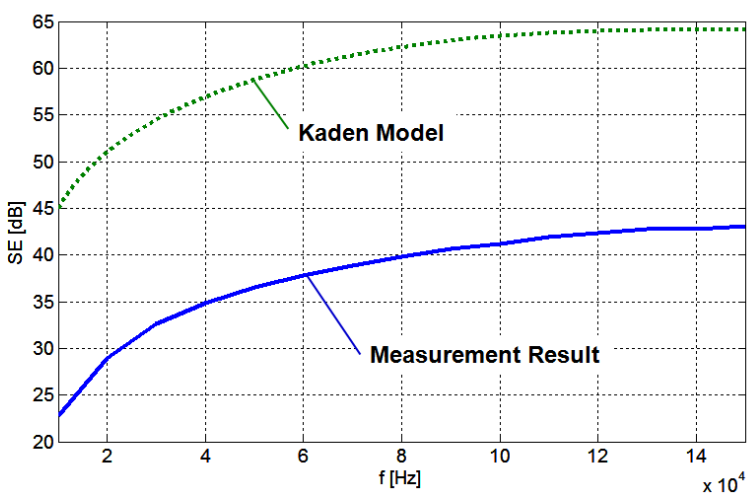

Fig. 14. Measured and calculated (Kaden, 1959) shielding effectiveness (SE) of a vertical oriented wire mesh enclosure.

\subsection{Comparison with analytical model}

For the comparison with the analytical model of Kaden, the results of the vertical shielding effectiveness measurement were used. There is an even better approach to the parallelplate-shield. The comparison with the analytical model and the measurement results of the vertical oriented enclosure is shown in Fig. 14. In the literature (for example Wolfsperger (2008); page 207) it was also reported, that the theoretical calculated shielding effectiveness will be according to experience maximal $10-20 \mathrm{~dB}$ higher than the practical reached values. This could also be observed for the measurement results presented in this paper. The measured results and the model of Kaden are showing an almost constant deviation for the frequency range to be covered (approximately $22 \mathrm{~dB}$ ). The model uses the exact material parameters of the wire mesh enclosure (mesh size: $\sim a_{s}=364 \mu \mathrm{m}$, wire radius: $\left.\sim r_{w}=100 \mu \mathrm{m}\right)$. This deviation is mainly due to:

\section{Geometric properties of the model and the enclosure}

\section{Electrical connections between the plates and the sides}

The geometric properties differ. The model is based on a conductor loop and the wire mesh enclosure is a fully shielded box (6 sides for shielding). Subsequently the aspect ratio of the model is assumed to be $\left|y_{0}\right|>>2 \cdot x_{0}$ and for the wire mesh enclosure $y_{0}=2 \cdot x_{0}$. The effective area of the conductor loop from Kaden is even larger than the effective area of the wire mesh enclosure and the induced voltage depends on the size of the area, which interacts the magnetic field. The geometric dimensions of the shield, like the effective shielding area are not considered in the model from Kaden. Just the distance between the plates has been considered. The increasing resistance by raising the distance between the plates is also not considered in the model. Although the electrical connections between the plates of the wire mesh enclosure are not perfect. 


\section{Conclusions}

A simple and reproducible measurement method for the magnetic shielding effectiveness in frequency range from $10 \mathrm{kHz}$ to $150 \mathrm{kHz}$ of small enclosures was presented in this paper. The results have shown, that the magnetic shielding effectiveness is also a function of the area size, where the incident field is interacting (eddy currents). This effect could be used to reduce the electromagnetic interference of cables near sensitive circuits in enclosures. The comparison with an analytical model (Kaden, 1959) has shown, that the physical behaviour is described correctly. The deviation to the measured results is approximately $22 \mathrm{~dB}$. The main reason for the difference are the geometric assumptions, such as no consideration of the exact shield geometry in the model from Kaden. Another reason could be the quality of the electrical connection between the sides of the wire mesh enclosure. With a better electrical connection (low-ohmic) improved shielding effectiveness could be achieved.

\section{Outlook}

As shown in conclusions, the general physical behaviour of the analytical model in comparison to the measurements is described suitable. Nevertheless there is a deviation by $\sim 22 \mathrm{~dB}$ for the vertical position of the enclosure in the frequency range. Therefore, the next step is the comparison of the measurement results (vertical and horizontal position) with results gained by numerical field calculations. Based on a numerical field calculations it will be possible to add a correction term to the used analytical model based on an empirical approach. This correction term will describe the influence of the geometrical properties in a better way. As shown in this paper, the magnetic shielding effectiveness of an enclosure depends on the size of the area, where the incident magnetic field vector is oriented perpendicular. Also, sharp edges and corners (see corner effect, Kaden, 1959) have an influence on the the shielding effectiveness and need to be analyzed within the next steps. The connection of the enclosure parts have also an impact to increase or decrease the shielding effectiveness.
Acknowledgements. This contribution was developed within the scope of the project EM4EM (Electromagnetic Reliability and Electronic Systems for Electro Mobility - Subproject: Entwurfs- und EMZ-Messmethodik für EMZ-Analysen auf EVGesamtsystemebene) which is funded by the BMBF (Bundesministerium für Bildung und Forschung) under the grant number 16M3092A. The responsibility for this publication is held by the authors only.

\section{References}

DIN EN 61000-5-7; Elektromagnetische Verträglichkeit (EMV) - Teil 5-7: Installationsrichtlinien und Abhilfemaßnahmen; Schutzarten durch Gehäuse gegen elektromagnetische Störgrößen (EM-Code), Beuth Verlag, 2001.

CISPR 25; Vehicles, boats and internal combustion engines-Radio disturbance characteristics-Limits and methods of measurement for the protection of on-board receivers, Edition 3, 2007.

Casey, K.: Electromagnetic shielding behavior of wire-mesh screens, IEEE Transactions on Electromagnetic Compatibility, 30, 298-306, doi:10.1109/15.3309, 1988.

Hillmer, C., Mayer, M.-O., and Reinhardt, U.: EMV von Elektro-Hybridfahrzeugen, in: Vortrag der 5. GMM-Fachtagung München, 2009.

Kaden, H.: Wirbelströme und Schirmung in der Nachrichtentechnik, Springer-Verlag, 2. Auflage, 1959.

Kistenmacher, P., Kimmel, M., and Schwab, A.: Schirmdämpfung inhomogener Metallgehäuse beliebiger Kontur im quasistationären Magnetfeld, Electrical Engineering 78 Springer-VerlagVerlag, 339-344, 1995.

Michel, M.: Leistungselektronik: Einführung in Schaltungen und deren Verhalten, Springer-Verlag, 5. Auflage, 2011.

Schwab, A. and Kürner, W.: Elektromagnetische Verträglichkeit, VDI-Buch, Springer-Verlag, 6. Auflage, 2010.

Wolfsperger, H.: Elektromagnetische Schirmung: Theorie Und Praxisbeispiele, VDI-Buch, Springer-Verlag, 2008. 